\title{
Projeção da mortalidade e internações hospitalares na rede pública de saúde atribuíveis à poluição atmosférica no Estado de São Paulo entre 2012 e 2030
}

\author{
Cristina Guimarães Rodrigues* \\ Evangelina da Motta Pacheco Araújo Vormittag ${ }^{\star \star}$ \\ Julia Affonso Cavalcante ${ }^{\star \star \star}$ \\ Paulo Hilário Nascimento Saldiva ${ }^{\star \star \star \star}$
}

No Brasil, os padrões nacionais de qualidade do ar são da década de 1990, desatualizados em relação aos internacionais, o que dificulta um controle eficaz dos níveis de poluição e contribui para a continuidade de mortes e internações. A fim de quantificar o montante de desfechos desfavoráveis de saúde em decorrência da concentração de material particulado no Estado de São Paulo, esse trabalho tem como objetivo realizar projeções de mortes, internações e gastos com internações públicas atribuíveis à poluição atmosférica, para o período de 2012 a 2030. Para isso foram considerados distintos cenários de poluição por material particulado fino $\left(\mathrm{MP}_{2,5}\right)$, tomando-se como referência o ano-base 2011. Os resultados mostram que, mantendo o mesmo nível de poluição observado em 2011, haverá um total de mais de 246 mil óbitos por todas as causas entre 2012 e 2030, cerca de 953 mil internações hospitalares públicas e um gasto público estimado em internações de mais de $\mathrm{R} \$ 1,6$ bilhão. A magnitude dos resultados aponta para a necessidade de implementação de medidas mais rigorosas para o controle da poluição do ar, formas alternativas de energia limpa de transporte, entre outras ações, como forma de reduzir os danos à saúde da população e os gastos governamentais.

Palavras-chave: Poluição do ar. Saúde pública. Estado de São Paulo. Material particulado.

\footnotetext{
* Fundação Instituto de Pesquisas Contábeis, Atuariais e Financeiras (Fipecafi), São Paulo-SP, Brasil (guimaraes.cristina@ gmail.com).

** Instituto Saúde e Sustentabilidade, São Paulo-SP, Brasil (vanjav@saudeesustentabilidade.org.br).

${ }^{* * \star}$ Faculdade de Filosofia, Letras e Ciências Humanas, da Universidade de São Paulo (FFLCH/USP), São Paulo-SP, Brasil (julia.affonsoc@gmail.com).

${ }_{\star \star \star \star}$ Faculdade de Medicina da Universidade de São Paulo (USP), São Paulo-SP, Brasil (pepino@usp.br).
} 


\section{Introdução}

Nas duas últimas décadas tem havido um intenso debate mundial sobre as questões socioambientais e as externalidades negativas provenientes das atividades antrópicas que provocam a diminuição da qualidade de vida, principalmente, nas cidades. Entre os desequilíbrios urbano-ambientais da contemporaneidade que atingem a saúde humana, está a poluição atmosférica. A literatura mundial tem corroborado o fato de a variação tóxica dos poluentes no ambiente afetar a saúde de diferentes maneiras e níveis de gravidade. Por exemplo, a poluição do ar está relacionada à redução da expectativa de vida e ao aumento do risco de arritmias e infarto agudo do miocárdio, bronquite crônica e asma, doenças pulmonares obstrutivas crônicas (DPOC), obesidade, câncer do pulmão e depressão (DOCKERY et al., 1993; SALDIVA et al.,1995; POPE et al., 1995, 2002; BRAGA et al., 2001; CONCEIÇÃO et al., 2001; ANDERSON et al., 2004; FARHAT et al., 2005; PETERS, 2005; CANÇADO et al., 2006; LADEN et al., 2006; WHO, 2006; POPE; DOCKERY, 2006; NASCIMENTO et al., 2006; ULIRSCH et al., 2007; CAREY et al., 2013; DOMINICI et al., 2002).

Os grupos populacionais mais suscetíveis aos efeitos tóxicos da poluição do ar são crianças abaixo de cinco anos de idade, idosos e indivíduos portadores de doenças crônicas. As crianças, por exemplo, já estão submetidas aos efeitos adversos da poluição atmosférica antes mesmo de nascer. Estudos mostram que o aumento nos níveis de poluição atmosférica está associado ao crescimento no risco de mortalidade infantil (RITZ; WILHELM; ZHAO, 2006) e déficits no desenvolvimento pulmonar (SOFER et al., 2013) e ao aumento no acesso aos serviços de saúde em decorrência de doenças respiratórias como asma (McCORMACK et al., 2011). No Brasil, alguns estudos demonstram o retardo do crescimento intrauterino, menor peso ao nascer e maior mortalidade intrauterina e neonatal (PEREIRA et al., 1998; LIN et al., 2004; MEDEIROS et al., 2005).

A severidade dos efeitos da poluição sobre a saúde dos idosos decorre da fragilidade do sistema imunológico com o avanço da idade. Em geral, estudos mostram que a poluição atmosférica, principalmente aquela relacionada ao material particulado, está associada ao aumento da mortalidade em idosos e à maior frequência de internações hospitalares por problemas cardiovasculares e respiratórios por asma e DPOC (DOMINICI et al., 2002; WELLENIUS; SCHWARTZ; MITTLEMAN, 2006; BELL et al., 2006; BENTAYEB et al., 2012). Um dos maiores estudos realizados no Brasil a respeito do efeito da poluição sobre a saúde dos idosos foi desenvolvido por Saldiva et al. (1995). Este estudo procurou mostrar o efeito do aumento do material particulado sobre a mortalidade de pessoas com mais de 65 anos de idade, na Região Metropolitana de São Paulo. 0 estudo constatou que a elevação em $10 \mathrm{mg} / \mathrm{m}^{3}$ de material particulado estava associada a um crescimento na mortalidade geral de idosos em mais de $13 \%$, mostrando que a poluição do ar tem efeito significativo sobre a mortalidade de pessoas idosas.

Nesse contexto, o objetivo do presente trabalho é contribuir para o debate acerca dos efeitos da poluição atmosférica - especificamente o material particulado $\mathrm{MP}_{2,5}$ - sobre 
mortes, internações e custos da rede pública de saúde no Estado de São Paulo em uma perspectiva temporal. Os resultados apresentados têm como finalidade informar o gestor público o quanto se perde em vidas, saúde e recursos públicos ao adiar medidas que contribuam para a melhoria da qualidade do ar.

\section{Material particulado e saúde}

A Organização Mundial da Saúde (OMS) lista uma série de componentes tóxicos para a saúde relacionados à poluição do ar. Estes componentes podem tanto ser emitidos para a atmosfera, como dióxido de enxofre, monóxido de carbono e óxido de nitrogênio, quanto formados a partir de reações químicas, a exemplo do ozônio e material particulado (WHO, 2006).

Um dos poluentes com maior efeito sobre a saúde é o material particulado, que corresponde a uma mistura de componentes sólidos e líquidos que podem variar na composição, tamanho e de acordo com a fonte de emissão e condições meteorológicas presentes no ambiente. As partículas inaláveis, que possuem maior impacto sobre a saúde humana, podem ser definidas de maneira simplificada como $\mathrm{MP}_{10}$ (partículas inaláveis), aquelas

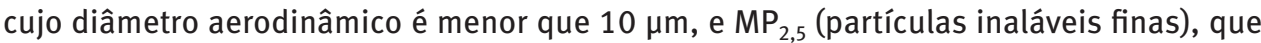
possuem diâmetro aerodinâmico inferior a $2,5 \mu \mathrm{m}$. 0 tamanho da partícula possui relação inversamente proporcional ao potencial de deposição no trato respiratório e os efeitos à saúde associados. Ou seja, partículas maiores podem ficar retidas na parte superior do sistema respiratório, enquanto as menores, $\mathrm{o} \mathrm{MP}_{2,5}$, atingem as vias respiratórias inferiores, podem alcançar os alvéolos e possuem a característica de transportar gases absorvidos em sua superfície até onde ocorre a troca gasosa nos pulmões (BRUNEKREEF; HOLGATE, 2002; LADEN et al., 2006).

Assim, a poluição por $\mathrm{MP}_{2,5}$ tornou-se um importante fator de risco para estudos epidemiológicos em saúde, uma vez que possuem as associações causais mais robustas entre a exposição de longo prazo ao poluente e a redução da expectativa de vida (DOCKERY et al., 1993; LIPFERT, 1984; POPE et al., 1995). O Global Burden of Disease, programa global de pesquisa investigativa da OMS que avalia a mortalidade e incapacidade por doenças graves, lesões e fatores de risco, estabeleceu em 2010 a poluição atmosférica por material particulado fino como o sexto maior fator de risco para a mortalidade prematura global. Para efeito de comparação, a carga da doença atribuída ao $\mathrm{MP}_{2,5}$ é maior do que outras ameaças à saúde global bem reconhecidas, tais como malária e HIV-AIDS combinadas (APTE et al., 2015; IHME, 2012).

A Agência Internacional de Pesquisas sobre o Câncer (IARC), vinculada à Organização Mundial de Saúde (OMS), anunciou, em 2013, a classificação da poluição do ar exterior (outdoor) e do poluente material particulado como substâncias carcinogênicas do Grupo I. Isso significa que o risco de desenvolver câncer de pulmão ou bexiga é significativamente maior em pessoas expostas à poluição atmosférica (IARC, 2013). Revisões sistemáticas sobre o efeito do material particulado e câncer de pulmão foram realizadas a partir do relatório do IARC para corroborar os efeitos nocivos da poluição sobre essa doença (HAMRA et al., 
2014). Além disso, em 2015, a OMS divulgou a perda precoce de cerca de oito milhões de vidas no mundo pela poluição do ar. Desses, cerca de 3,7 milhões deveram-se à poluição do ar externa, o que representa $46 \%$ de todas as fontes de poluentes relacionadas ao ar. Reconhecendo-a como uma ameaça cada vez maior à saúde pública global, em relatório oficial da 68 assembleia Mundial da Saúde, em maio de 2015, a OMS concluiu que a redução da poluição atmosférica pode se tornar um indicador sanitário das políticas dos objetivos do desenvolvimento sustentável pós-2015 (WHO, 2015). Neste documento, a OMS solicita aos países-membro que tomem medidas emergenciais de controle efetivo da poluição, uma vez que a poluição do ar passa a ser líder ambiental para riscos em saúde.

Diretrizes globais sobre poluição do ar e legislação nacional

Os padrões de poluição do ar definidos pela OMS, em 2005, são orientadores para a criação de padrões regionais do nível de poluentes. Para a concentração de $\mathrm{MP}_{2,5}$, é estabelecido como critério aceitável - o menor efeito sobre a saúde - o máximo de $10 \mu \mathrm{g} / \mathrm{m}^{3}$ anual e $25 \mu \mathrm{g} / \mathrm{m}^{3}$ de média diária (WHO, 2006), mas os países podem adotar distintos padrões, que variam de acordo com as especificidades locais. Nos Estados Unidos, por exemplo, a Environmental Protection Agency (EPA), responsável pela proteção ambiental, define um nível máximo de $15 \mu \mathrm{g} / \mathrm{m}^{3}$ e $35 \mu \mathrm{g} / \mathrm{m}^{3}$ como as médias anuais e diárias de $\mathrm{MP}_{2,5}$ (EPA, 2013). A European Environmental Agency (EEA) fixa como padrão máximo a média anual de $25 \mu \mathrm{g} / \mathrm{m}^{3}$ de $\mathrm{MP}_{2,5}$, e não estabelece padrões diários de emissão de poluentes.

Vahlsing e Smith (2012) realizaram uma revisão global sobre os padrões de qualidade de ar para $\mathrm{MP}_{10}$, por meio de questionários aplicados em 96 países, que representam $84 \%$ da população no mundo, mostrando que $72 \%$ deles possuíam padrão de medida diária para $\mathrm{MP}_{10}$. A média do valor diário do padrão encontrada entre os países foi $98 \mu \mathrm{g} / \mathrm{m}^{3}$, bem acima do preconizado pela OMS $\left(50 \mu \mathrm{g} / \mathrm{m}^{3}\right)$ e, no Brasil, abaixo do padrão estabelecido em 1990, pela Resolução Conama 03/90, de $150 \mu \mathrm{g} / \mathrm{m}^{3}$. Além disso, o Brasil é tido como o penúltimo país a ter alterado o padrão, na década de 1990, comparado aos outros países que vêm estabelecendo a atualização de seus padrões desde então (VAHLSING; SMITH, 2012). A Resolução, estabelecida há 25 anos, apenas fixa o limite para $\mathrm{MP}_{10}$. Em 2013, o Conselho Estadual do Meio Ambiente do Estado de São Paulo (Consema) definiu novos critérios para $\mathrm{MP}_{10}$ e estabeleceu o padrão para $\mathrm{MP}_{2,5}$, até então inexistente no Brasil. 0 Decreto n. 59.113/2013 estabelece os limites anual e diário para concentração de $\mathrm{MP}_{2,5}$ em $20 \mu \mathrm{g} / \mathrm{m}^{3}$ e $60 \mu \mathrm{g} / \mathrm{m}^{3}$, respectivamente.

Em 2014, o Conselho Nacional do Meio Ambiente (Conama) implementou um grupo de trabalho para a revisão da Resolução Conama 03/90 (BRASIL, 1990). A proposta desse grupo de trabalho era determinar a alteração dos padrões de qualidade do ar em etapas, até atingir os níveis propostos pela OMS. Porém, não foi determinado prazo para que essas etapas entrassem em vigor e não houve um consenso após um ano de discussões, o que inviabilizou a implementação da resolução. 
Com o intuito de embasar a defesa do estabelecimento de prazo na discussão sobre o risco de saúde a que a população seria submetida aos níveis atuais do poluente MP nos próximos anos, decidiu-se pela realização do presente estudo. Além disso, o Estado de São Paulo concentra grande parte da população brasileira, sendo um dos estados mais populosos e urbanizados, com cerca de $1 / 5$ da população do país e uma taxa de urbanização de quase $96 \%$, segundo o Censo Demográfico 2010, do Instituto Brasileiro de Geografia e Estatística (IBGE). Dos 645 municípios do Estado, apenas 42 monitoram a qualidade do ar, com 86 estações de monitoramento, o que representa aproximadamente $34 \%$ das estações existentes no país (VORMITTAG et al., 2014). Logo, é um estado com maior disponibilidade de informações para o desenvolvimento de estudos sobre o efeito da poluição sobre a saúde.

O objetivo geral desse trabalho é estimar, para o período 2012-2030, o efeito do $\mathrm{MP}_{2,5}$ sobre mortes e internações da rede pública de saúde no Estado de São Paulo para causas selecionadas. Utilizou-se o padrão estabelecido pela OMS em substituição ao padrão nacional, como subsídio para a mudança da política vigente no país. Além disso, os resultados apresentados têm como finalidade informar ao gestor público o quanto se perde em vidas, saúde e recursos públicos ao adiar medidas que contribuam para a melhoria da qualidade do ar.

\section{Metodologia}

Nessa seção, apresenta-se o método de estimação do número de mortes, internações e custos das internações da rede pública de saúde atribuíveis ao material particulado $\mathrm{MP}_{2,5}$ no Estado de São Paulo, entre 2012 e 2030, para causas selecionadas, bem como o baseline utilizado na projeção. 0 cálculo da carga de doenças atribuíveis à poluição segue metodologia proposta pela Organização Mundial de Saúde, segundo comunicação direta com o grupo do Environmental Burden of Disease da mesma instituição e diretrizes sobre qualidade do ar em 2005 (OSTRO, 2004; WHO, 2006).

\section{Baseline e cenários de poluição}

0 ano de 2011 foi escolhido como baseline para as projeções tanto de mortalidade quanto de internações hospitalares. A seguir, apresentam-se as causas de mortalidade e morbidade consideradas no estudo.

- Mortalidade (CID 10 especificada entre parênteses)

- Mortalidade geral: capítulo I a XVI da CID 10 para todos os grupos etários.

- Câncer: neoplasias malignas do aparelho respiratório e órgãos intratorácicos (C30 - C39) para a população acima de 40 anos.

- Doenças cardiovasculares: doenças cerebrovasculares (160-169) e doença isquêmica do coração (120-125) para a população de 40 anos ou mais. 
- Doença respiratória em crianças: pneumonia e influenza (J09-J18) e outras infecções agudas das vias aéreas inferiores (J20-J22) para crianças com menos de cinco anos.

- Doença respiratória em idosos: pneumonia e influenza (J09-J18) e doenças crônicas das vias aéreas inferiores (J40-J47) para a população acima de 60 anos.

- Morbidade hospitalar (CID 10 para morbidade especificada entre parênteses)

- Câncer: neoplasia maligna de traqueia, brônquios e pulmões (C33-C34) para a população acima de 40 anos.

- Doenças cardiovasculares: acidente vascular cerebral não especificado hemorrágico ou isquêmico (164), hemorragia intracraniana (160-162), infarto agudo do miocárdio (121-122), infarto cerebral (163) e outras doenças isquêmicas do coração (120, 123-125), para a população de 40 anos ou mais.

- Doença respiratória em crianças: pneumonia (J12-J18) para crianças com menos de cinco anos.

- Doença respiratória em idosos: bronquite, enfisema e outras doenças pulmonares obstrutivas crônicas (J40-J44), asma (J45-J46) e pneumonia (J12-J18) na população acima de 60 anos.

Os dados de mortalidade foram obtidos do Sistema de Informações de Mortalidade (SIM), disponibilizados pelo Ministério da Saúde, Secretaria de Vigilância à Saúde (MS/ SVS), e os de morbidade hospitalar são oriundos do Sistema de Informações Hospitalares (SIH/SUS), ambos para 2011.

Para a poluição, o baseline corresponde à média anual ${ }^{1}$ do $\mathrm{MP}_{2,5}$ no Estado de São Paulo, calculada a partir dos dados da Companhia Ambiental do Estado de São Paulo Cetesb, cujo valor em 2011 era de $22,48 \mu \mathrm{g} / \mathrm{m}^{3}$. Os valores de $\mathrm{MP}_{2,5}$ foram obtidos a partir dos dados de monitoramento de qualidade do ar de $\mathrm{MP}_{10}$, considerando a fração de $0,6 \%$ (CETESB, 2014). Os cenários de poluição (concentração do material particulado $\mathrm{MP}_{2,5}$ ) considerados no estudo são:

- estacionário: neste cenário, supomos que os níveis de poluição por MP de 2011 se manterão constantes no futuro;

- aumento de 5\% de $\mathrm{MP}_{2,5}$ até 2030: o equivalente a +0,003\% ao ano, entre 2012 e 2030;

- redução de 5\% de $\mathrm{MP}_{2,5}$ até 2030: o equivalente a -0,003\% ao ano, entre 2012 e 2030.

\footnotetext{
${ }^{1}$ Esse valor médio foi aplicado ao total da população do Estado de São Paulo, considerando os municípios com mais de uma estação de monitoramento ou aqueles sem nenhuma estação. Das 42 estações de monitoramento no Estado, mais da metade (24 estações) apresentou níveis médios de material particulado situados entre 20 e $25 \mu \mathrm{g} / \mathrm{m}^{3}$ em 2011. Essas estações captam tanto municípios com grande contingente populacional, como São Paulo, quanto aqueles de menor atividade industrial ou menor contingente populacional, como Paulínia. Nesse sentido, embora não seja possível captar os níveis de poluição de diferentes grupos populacionais ou de todos os municípios, utilizamos como pressuposto que a média do Estado fornece uma estimativa do que poderia ser observado em todo o Estado, pois as estações são representativas de municípios com diferentes tipos de atividade econômica e distintos tamanhos populacionais. Além disso, os 29 municípios monitorados concentram $86 \%$ da população do Estado, exposta à emissão de material particulado por diferentes fontes, como veículos automotores, fonte industrial, queima de cana-de-açúcar e ressuspensão de poeira.
} 


\section{Métodos de projeção}

A projeção da mortalidade e internações atribuíveis à poluição envolve os seguintes passos:

- projeção do número de mortes entre 2012 e 2030;

- estimativa do número de mortes atribuíveis à poluição atmosférica entre 2012 e 2030, a partir dos cenários de poluição considerados;

- projeção do número de internações hospitalares públicas entre 2012 e 2030;

- estimativa do número de internações atribuíveis à poluição atmosférica entre 2012 e 2030, a partir dos cenários de poluição considerados;

- projeção do gasto total com internações públicas, a partir da estimativa do número de internações atribuíveis à poluição obtida na projeção anterior.

A metodologia adotada para cada um dos componentes das projeções é apresentada a seguir.

Projeção de mortalidade

Para a projeção da mortalidade, consideramos que o número de óbitos por causa selecionada, entre 2012 e 2030, é dado pela multiplicação da proporção projetada de óbitos em cada ano pelo número total de mortes no mesmo período:

$$
O^{z, t}=\sum_{x}{ }_{n} O_{x}^{z, t_{*}}{ }_{n} O_{x}^{t}
$$

Onde:

$O^{z, t}=$ número de óbitos pela causa $z$ no ano $t$, correspondente a cada ano da projeção; ${ }_{n} P O_{x}^{z, t}=$ proporção de óbitos pela causa $z$ no ano $t$ para o grupo etário $x$ a $x+n$ (grupos etários quinquenais);

${ }_{n} O_{x}^{t}=$ total de óbitos no ano $t$ para o grupo etário $x$ a $x+n$ (grupos etários quinquenais).

0 total de óbitos em cada grupo etário $\left(O^{z, t}\right)$ foi obtido das projeções de mortalidade do IBGE (Revisão 2013) para todo o Estado de São Paulo. Para a projeção da proporção de óbitos em cada causa e grupo etário entre 2012 e $2030\left({ }_{n} P O_{x}^{z, t}\right)$, adotamos a suposição de que a proporção de óbitos por causa e grupo etário seguiria a tendência observada entre 2000 e 2011 para este indicador. A projeção da proporção de óbitos seguiu a metodologia adotada em Rodrigues et al. (2013):

$$
{ }_{n} P O_{x}^{z, t+1}=\frac{1}{2} \ln \left(\frac{1-{ }_{n} P O_{x}^{z, t}}{{ }_{n} P O_{x}^{z, t}}\right) *\left[1+{ }_{n} w_{x} *((t+1)-t)\right]
$$

Onde:

${ }_{n} w_{x}=$ variação anual na proporção de óbitos por causa e grupo etário $x$ a $x+n$, dada por: 


$$
{ }_{n} w_{x}=\frac{\left(\frac{{ }_{n} P O_{x}^{2011}}{{ }_{n} P O_{x}^{2000}}-1\right)}{11}
$$

Com a projeção do número de óbitos, o passo seguinte foi estimar o número de mortes atribuíveis à poluição, de acordo com a metodologia proposta por WHO (2006). Nesta metodologia, estima-se a fração atribuível à poluição, a partir da função do risco relativo e, então, é estimado o número atribuível de mortes.

A função de risco relativo utilizada corresponde à função:

$$
R R=\left[\frac{\left(M_{2,5}^{\text {Observado }}+1\right)}{\left(P M_{2,5}^{\text {Padrão }}+1\right)}\right]^{\text {beta }}
$$

0 padrão empregado no cálculo do risco relativo corresponde a $10 \mu \mathrm{g} / \mathrm{m}^{3}$, critério utilizado pela OMS para médias anuais de $\mathrm{MP}_{2,5}$. A fração atribuível é dada por:

$$
F A=\frac{(R R-1)}{R R}
$$

O número atribuível de mortes pela causa $z$ no tempo $t$, decorrente da poluição, então, é dado por:

$$
{ }_{n} O_{x}^{\text {Atribuivel, } z, t}=O^{z, t} * F A
$$

Projeção do número de internações hospitalares públicas

A projeção de internações segue o padrão usual de projeção, em que as taxas de internação por causas e grupos etários em cada período são multiplicadas pela população em cada período e grupo etário:

$$
I^{z, t}=\sum_{x}{ }_{n} T I_{x}^{z, 201{ }^{*}} P_{x}^{t}
$$

Onde:

$I^{z, t}=$ número de internações pela causa $z$ no ano $t$, correspondente a cada ano da projeção;

${ }_{n} T I_{x}^{z, 2011}=$ taxa de internação pela causa $z$ em 2011 para o grupo etário $x$ a $x+n$ (grupos etários quinquenais);

${ }_{n} P_{x}^{t}=$ número de pessoas no ano $t$ para o grupo etário $x$ a $x+n$ (grupos etários quinquenais).

O número projetado de internações segue a metodologia de taxas fixas (STRUNK et al., 2006), em que se leva em conta apenas o efeito das mudanças demográficas sobre as taxas. Não foi possível projetar as taxas considerando a tendência histórica, uma vez que as taxas de internação por grupo de causas não apresentaram regularidade ao longo dos anos, não sendo possível verificar tendência. Logo, para todos os anos da projeção, 
consideraram-se a taxa de internação de 2011 e a população projetada pelo IBGE (2013) por grupo etário.

O risco relativo e a fração atribuível foram calculados a partir das equações (4) e (5). O beta utilizado para o cálculo do risco relativo pode ser visto no Quadro 1.0 número de internações atribuíveis em cada causa foi dado por:

$$
{ }_{n} I_{x}^{\text {Atribuível,z,t }}=I^{z, t} * F A
$$

Projeção do gasto total com internações hospitalares públicas

Para a projeção de gastos, o valor médio das internações é multiplicado pelo número projetado de internações atribuíveis à poluição em cada ano, resultantes das estimativas obtidas em (2):

$G^{z, t}=\sum_{x} G M e_{x}^{z, 2011 *} I_{x}^{\text {Atribuivel }, z, t}$

0 gasto médio com internações para cada uma das causas foi mantido fixo no mesmo nível de 2011. Os gastos projetados são a preços de 2011.

O parâmetro beta utilizado para cada uma das causas de morte e internação é apresentado no Quadro 1.

QUADRO 1

Parâmetro beta utilizado no cálculo do risco relativo de mortalidade e morbidade, para cada variação em $10 \mathrm{mg} / \mathrm{m}^{3}$ de material particulado $\mathrm{MP}_{2,5}$

\begin{tabular}{llcl}
\hline \multicolumn{1}{c}{ Desfecho em saúde } & \multicolumn{1}{c}{ Causa } & Beta & \multicolumn{1}{c}{ Fonte } \\
\hline \multirow{3}{*}{ Mortalidade } & Todas as causas & 0,06 & WHO (2005) \\
& Cardiovascular & 0,155 & Pope et al. (2002) \\
& Câncer de pulmão & 0,232 & Pope et al. (2002) \\
& Doenças respiratórias em idosos & 0,155 & Pope et al. (2002) \\
& Doenças respiratórias em crianças & 0,091 & Pope et al. (2002) \\
\hline \multirow{2}{*}{ Internações hospitalares } & Cardiovascular & 0,18 & Pope (2004) \\
& Câncer de pulmão & 0,40 & Nielsen (2013) \\
& Doenças respiratórias em idosos & 0,31 & Cançado (2006) \\
& Pneumonia em crianças & 0,214 & Cançado (2006) \\
\hline
\end{tabular}

\section{Resultados}

A Tabela 1 traz o somatório das mortes, número de internações e gasto total com internações entre 2011 e 2030 atribuíveis à poluição para cada um dos cenários considerados, além de informações para 2015 e 2025 para análise de tendência dos números absolutos. Os resultados mostram que, entre 2011 e 2030, no Estado de São Paulo, o total de mortes atribuíveis à poluição chegaria a mais de 246 mil óbitos no cenário estacionário de poluição e ocorreriam quase 918 mil internações apenas por causas cardiovasculares, respiratórias e neoplasias selecionadas em grupos etários mais suscetíveis. Considerando os distintos 
cenários para a mortalidade geral, espera-se que, em 20 anos, o total de óbitos varie entre 236 mil, para uma redução de 5\% no nível da poluição até 2030 , e 256 mil, em caso de aumento da poluição em $5 \%$.

A mortalidade geral atribuível ao material particulado (que abrange todos os grupos etários) no Estado de São Paulo apresentou crescimento de $96 \%$ no período de análise, com 9.384 mortes em 2011 e uma projeção de 18.407 mortes para 2030 (Tabela 1). 0 número de mortes em 2011 representa cerca de 3,9\% do total de óbitos no Estado de São Paulo, valor inferior ao encontrado pelo estudo de Carga de Doenças (WHO, 2014), que aponta um percentual de mortes atribuíveis à poluição de $7 \%$ em 2012, considerando a média mundial, porém dentro dos limites esperados, entre 3\% e 8\% de mortes atribuíveis à poluição (WHO, 2006, 2014).

TABELA 1

Projeção do total de mortes, internações hospitalares da rede pública de saúde e gastos com internações, por cenários considerados, segundo causas atribuíveis ao material particulado $\mathrm{MP}_{2,5}$ Estado de São Paulo - 2011-2030

\begin{tabular}{|c|c|c|c|c|c|}
\hline \multirow[t]{2}{*}{ Causas } & \multicolumn{3}{|c|}{ Cenário estacionário } & \multirow{2}{*}{$\begin{array}{c}\text { Aumento de } \\
5 \% \text { de } \mathrm{MP}_{2,5} \\
\text { até } 2030 \\
2011-2030\end{array}$} & \multirow{2}{*}{$\begin{array}{c}\text { Redução de } \\
5 \% \text { de } \mathrm{MP}_{2,5} \\
\text { até } 2030 \\
2011-2030\end{array}$} \\
\hline & 2015 & 2030 & $2011-2030$ & & \\
\hline \multicolumn{6}{|l|}{ Óbitos } \\
\hline Todas as causas & 10.193 & 18.407 & 246.375 & 256.515 & 236.198 \\
\hline Neoplasias & 1.126 & 2.182 & 28.248 & 29.347 & 27.139 \\
\hline Doenças cardiovasculares & 5.469 & 9.185 & 128.520 & 133.560 & 123.442 \\
\hline Doenças respiratórias em crianças & 38 & 23 & 596 & 615 & 986 \\
\hline Doenças respiratórias em idosos & 3.026 & 8.100 & 88.647 & 92.400 & 84.866 \\
\hline \multicolumn{6}{|l|}{ Internações hospitalares } \\
\hline Neoplasias & 1.220 & 1.929 & 29.592 & 30.595 & 28.571 \\
\hline Doenças cardiovasculares & 14.570 & 23.366 & 354.970 & 368.182 & 341.648 \\
\hline Doenças respiratórias em idosos & 16.387 & 31.335 & 430.365 & 446.064 & 414.452 \\
\hline Doenças respiratórias em crianças & 7.294 & 6.239 & 138.050 & 142.434 & 133.626 \\
\hline
\end{tabular}

\section{Gasto com internações} hospitalares (R\$ de 2011)

Neoplasias

$\begin{array}{lll}1.257 .663 & 1.959 .926 & 1.098 .685 .456\end{array}$

1.139.432.980 1.057.598.040

Doenças cardiovasculares

Doenças respiratórias em idosos

Fonte: Ministério da Saúde/Secretaria de Vigilância em Saúde. Sistema de Informações de Mortalidade (2012); Sistema de Informações Hospitalares (2012). IBGE. Projeções populacionais e de mortalidade (2013).

Em relação às internações hospitalares públicas, foram consideradas distintas causas de morbidade, de modo que representassem aquelas com estudos comprovados do efeito do material particulado sobre a saúde e respectivas estimativas de concentração-resposta. Essas causas correspondem a neoplasias, doenças respiratórias e doenças cardiovasculares, e em populações mais suscetíveis ao efeito da poluição, como crianças e idosos. Como esperado, as doenças cardiovasculares, consideradas a principal causa no país tanto em 
mortalidade quanto em morbidade, também figuram como as de maior efeito decorrente da poluição. Considerando apenas o cenário estacionário da poluição, as internações devido a essa causa variariam de 36.146 a 62.689, entre 2011 e 2030, e o gasto (a valores de $\mathrm{R} \$ 2011$ ) passou de $\mathrm{R} \$ 40$ milhões para $\mathrm{R} \$ 71$ milhões, nesse mesmo período. 0 valor total de internações ao ano ultrapassa R \$ 1 milhão, o que significa, em 20 anos, um dispêndio estimado de mais de $R \$ 1,5$ bilhão a preços de 2011.

Em relação à tendência de mortalidade e morbidade atribuíveis à poluição no período analisado, a Tabela 1 mostra o número de eventos em saúde associados ao material particulado para 2015 e 2030. Observa-se que há uma tendência de crescimento de mortes e internações para todas as causas analisadas, exceto para doenças do aparelho respiratório em crianças, cuja redução é explicada pela queda da fecundidade no Estado de São Paulo. Entre 2000 e 2010, houve um decréscimo populacional para o grupo de 0 a 4 anos no Estado da ordem de 516.792 crianças.

Os Gráficos 1, 2 e 3 apresentam projeção da participação relativa e do total de mortes, morbidade e valor da internação, respectivamente, segundo as causas atribuíveis à poluição pelo material particulado $\mathrm{MP}_{2,5}$, para períodos quinquenais entre 2015 e 2030, no cenário estacionário. Em relação à mortalidade (Gráfico 1), observa-se que, no período de projeção, os óbitos por doenças cardiovasculares representam o maior percentual de mortes entre todas as causas. Embora o montante de mortes não tenha decrescido em números absolutos, com um aumento esperado de 5.469 em 2015 para 9.185 em 2030, sua importância no conjunto das quatro causas decresce ao longo dos anos, chegando a representar menos da metade do total de mortes em 2030. Apenas as doenças do aparelho respiratório em adultos tiveram maior participação relativa, passando de 31\% em 2015 para quase $42 \%$ em 2030 . No contexto do rápido crescimento populacional para os grupos etários de idosos, o número esperado de mortes por causas respiratórias nesse grupo quase triplica, passando de cerca de 3 mil mortes em 2015 para mais de 8 mil em 2030.

As neoplasias atribuíveis à poluição apresentam participação reduzida no total das mortes no período, segundo as projeções realizadas. Essa causa de morte responde por cerca de $11 \%$ do total de óbitos ao longo de todo o período de projeção. A mortalidade por doenças respiratórias em crianças registra o menor percentual entre as causas (menos de $1 \%$ do total), além de redução no período. Diferente das outras causas, porém, verifica-se decréscimo em números absolutos nas mortes para esse grupo etário.

Já as internações atribuíveis à poluição por doenças do aparelho respiratório em crianças possuem representatividade maior no conjunto das internações (mais de $18 \%$ em 2015), embora sua participação decresça ao longo do tempo (Gráfico 2). Como as projeções consideraram um cenário de taxa de internação constante ao longo do tempo, essa redução reflete tanto o decréscimo populacional para esse grupo quanto o aumento da representatividade das doenças do aparelho respiratório em idosos. Para essa causa entre os idosos, o montante de internações atribuíveis à poluição passou de quase $42 \%$ para cerca de $50 \%$ entre 2015 e 2030 . A participação relativa das neoplasias permane- 
ceu praticamente inalterada, enquanto as doenças do aparelho circulatório aumentaram pouco, de 36,9\% em 2015 para 37,2\% em 2030, embora em números absolutos tenha praticamente dobrado (de 12.623 para 23.366, entre 2011 e 2030). 0 total de internações para essas quatro causas praticamente dobra no período, de mais de 39 mil internações em 2015 para quase 63 mil em 2030, no cenário estacionário.

\section{GRÁFICO 1}

Projeção da participação relativa e do total de mortes, no cenário estacionário, segundo causas atribuíveis ao material particulado $\mathrm{MP}_{2,5}$

\section{Estado de São Paulo - 2015-2030}

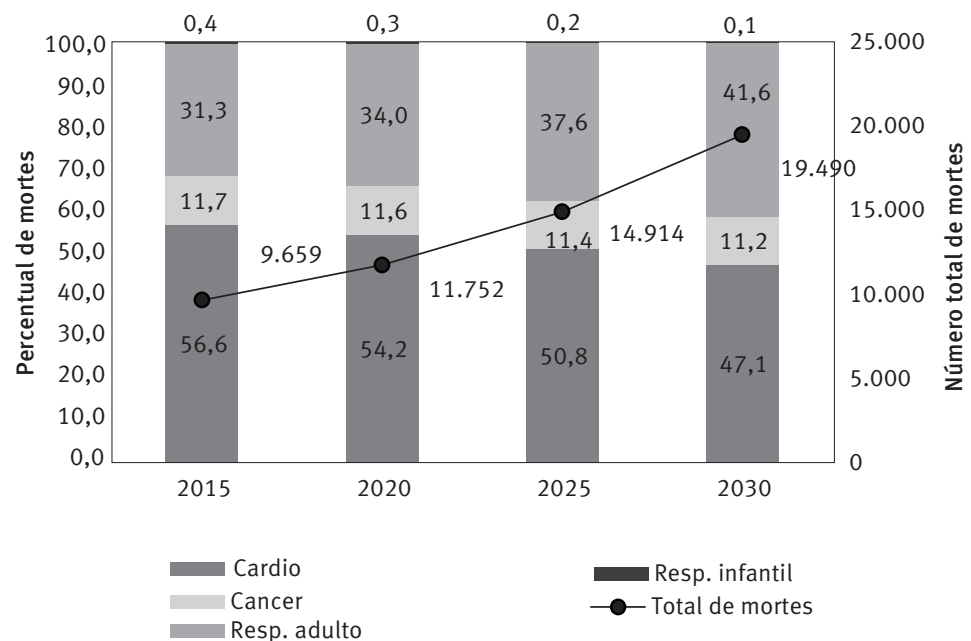

Fonte: Ministério da Saúde/Secretaria de Vigilância em Saúde. Sistema de Informações de Mortalidade (2012). IBGE. Projeções populacionais e de mortalidade (2013).

GRÁFICO 2

Projeção da participação relativa e do total das internações da rede hospitalar pública, no cenário estacionário, segundo causas atribuíveis ao material particulado $\mathrm{MP}_{2,5}$ Estado de São Paulo - 2015-2030

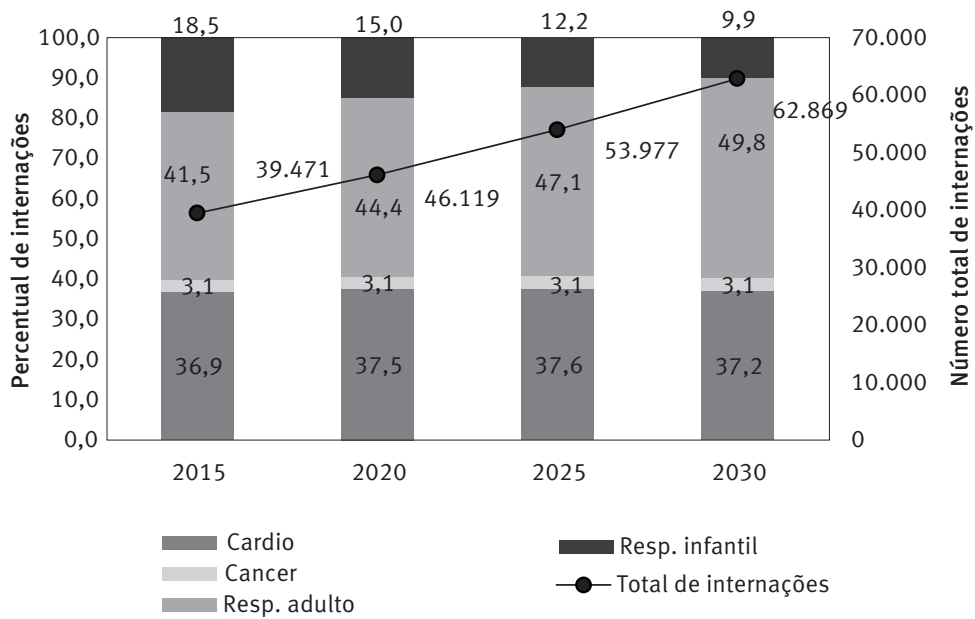

Fonte: Ministério da Saúde. Sistema de Informações Hospitalares (2012). IBGE. Projeções populacionais (2013). 
As doenças cardiovasculares, apesar de não representarem a maior participação relativa de internações entre as quatro causas consideradas, são responsáveis pelo maior percentual de gasto com internações, devido ao alto custo per capita de intervenções para o tratamento dessa causa (Gráfico 3). 0 percentual para essas doenças permaneceu praticamente constante entre 2015 e 2030, enquanto aquele referente às doenças respiratórias em idosos registrou aumento (cerca de $23 \%$ em 2015 e quase $28 \%$ em 2030) e o relativo a doenças respiratórias em crianças diminuiu de $10 \%$ para $5,5 \%$, no período. 0 total de gastos com internações hospitalares públicas atribuíveis à poluição pelo material particulado, apenas para essas quatro causas de internação, saltaria de $R$ \$ 70 milhões para $\mathrm{R} \$ 100$ milhões no cenário estacionário.

GRÁFICO 3

Projeção da participação relativa (projetadas) e do total de gastos com internações da rede hospitalar pública (1), no cenário estacionário, segundo causas atribuíveis ao material particulado $\mathrm{MP}_{2,5}$

Estado de São Paulo - 2015-2030

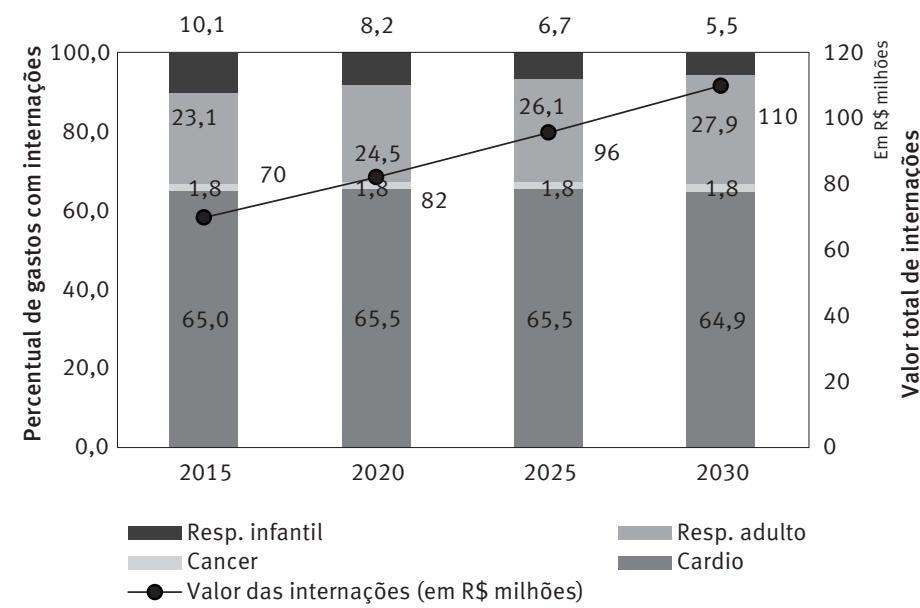

Fonte: Ministério da Saúde. Sistema de Informações Hospitalares (2012). IBGE. Projeções populacionais (2013). (1) $\mathrm{Em} \mathrm{R} \$$ de 2011.

\section{Discussão}

A inação em relação à política ambiental no mundo terá consequências graves sobre a saúde da população no futuro. Apenas no Estado de São Paulo, esperam-se quase 250 mil mortes até 2030 , caso os níveis de material particulado $\mathrm{MP}_{2,5}$ se mantenham nos mesmos níveis de 2011. 0 maior impacto ocorrerá para as doenças do aparelho circulatório e doenças respiratórias em idosos, visto que esse é o grupo que mais cresce na população e, juntamente com as crianças, o mais afetado pela poluição atmosférica. Neste cenário, o número de internações hospitalares somaria 1 milhão, com um gasto esperado de mais de $R \$ 1,5$ bilhão, ou o equivalente a quase US\$ 638 milhões. $^{2}$

\footnotetext{
$\overline{2}$ Foi considerada a taxa de câmbio média de R\$/US\$2,35 do ano de 2014, segundo dados do Ipeadata.
} 
Apesar disso, revisão sistemática realizada por Benmarhnia et al. (2014) mostrou que intervenções destinadas à melhoria da qualidade do ar não são equitativas. A população com maior poder aquisitivo apresenta maiores benefícios de intervenções voltadas à redução da poluição do ar, mostrando que tanto o nível dos poluentes quanto os benefícios advindos de intervenções no campo da legislação não são equânimes. Em São Paulo, a ausência de políticas de redução de poluentes em um futuro próximo provocará um aumento da poluição a partir de 2014. Desde 2009 observa-se uma linearidade nos níveis de emissão de MP no Estado, embora todas as cidades se situem com concentrações desse poluente superiores ao padrão preconizado pela OMS. As iniciativas do Proconve (Programa de Controle de Poluição Veicular), muito eficazes e fundamentais para a redução de poluentes no final da década de 1980 e início dos anos 1990, parecem não conseguir reduzir os níveis de poluentes no contexto atual.

Esse tipo de estudo é de suma importância em contextos urbanos com alta densidade demográfica e elevados níveis de poluição. Estudo realizado por Apte et al. (2015) mostra que a concentração espacial de mortalidade prematura decorrente da poluição $\left(\mathrm{MP}_{10} \mu \mathrm{g} / \mathrm{m}^{3}\right)$ é maior na Ásia, em função da interação entre alta densidade populacional, alta prevalência de doença e altos níveis de poluição. Embora o Global Health Observatory, da OMS, não tenha dados sobre o material particulado fino $\mathrm{MP}_{2,5}$ para anos recentes, informações sobre o $\mathrm{MP}_{10}$ mostram que Índia e China, por exemplo, figuram entre os países mais poluídos do mundo, com níveis de material particulado $\mathrm{MP}_{2,5}$ da ordem de 134 e 90 de $\mathrm{MP}_{10} \mu \mathrm{g} / \mathrm{m}^{3}$, respectivamente, quase 7 e 4,5 vezes o padrão médio anual recomendado pela OMS de $20 \mathrm{mg} / \mathrm{m}^{3}$.

Estudos similares de projeções foram realizados tanto para o contexto brasileiro quanto o mundial. Em uma análise prospectiva, Bell et al. (2005) mostraram que a diminuição de $10 \%$ de poluentes entre 2000 e 2020 na cidade de São Paulo acarretaria a redução de 138 mil visitas de crianças e jovens a consultórios, três mil visitas a prontos-socorros por doenças respiratórias e 817 mil ataques de asma. Estudo semelhante realizado por André et al. (2012) apontou que haveria um excesso de quase 14 mil mortes até 2040 e mais de 18 mil internações na rede pública de saúde em seis regiões metropolitanas brasileiras (São Paulo, Rio de Janeiro, Recife, Porto Alegre, Curitiba e Belo Horizonte), caso houvesse um atraso na implementação de políticas mais limpas de diesel. No presente estudo, um decréscimo da poluição da ordem de $5 \%$ em um período de 15 anos levaria a uma redução de 10.777 mortes, 1.021 internações hospitalares pelas quatro causas selecionadas no estudo e uma economia de mais de $R$ \$ 40 milhões em internações públicas em jovens.

O efeito da poluição sobre a saúde no futuro também foi estudado para outros países. Flachs et al. (2013), por exemplo, procuraram avaliar o efeito da dinâmica populacional sobre a mortalidade geral na população da Dinamarca decorrente da poluição pelo material particulado fino $\left(\mathrm{MP}_{2,5}\right)$, entre 2005 e 2030. Três cenários populacionais foram considerados: no primeiro, apenas o tamanho da população se altera (mantendo taxa de 
mortalidade e estrutura etária constantes); no segundo, tamanho e estrutura etária se alteram de acordo com as projeções populacionais (mantidas as taxas de mortalidade no nível de 2005); e, por fim, o último cenário modifica todos os componentes, incluindo uma redução esperada na taxa de mortalidade. 0 nível da poluição foi o mesmo de 2005 para todos os cenários avaliados. Assim como no Brasil, há um aumento esperado no número de mortes decorrentes da poluição, devido ao fato de que os grupos etários mais suscetíveis à mortalidade por poluição, como os idosos, apresentam também maiores taxas de crescimento populacionais. 0 estudo ressalta a importância de se considerar a dinâmica populacional na avaliação dos impactos futuros da poluição do ar sobre a saúde.

Estudo de Lelieveld et al. (2015) também destaca fatores demográficos, como crescimento da população residente em ambientes urbanos, para o aumento das mortes decorrentes da poluição. Os autores projetam a mortalidade por material particulado em várias regiões do mundo até 2050. Os resultados mostram que a taxa de mortalidade por poluição aumentaria de 5 mortes por 10.000 habitantes, em 2010 ( 2 milhões de mortes), para 7 mortes para cada 10.000 habitantes, em 2050 (cerca de 4,3 milhões de mortes em todo o mundo), sendo maior em ambientes urbanos.

No Brasil, apenas 1,7\% dos municípios possuíam estações de monitoramento em 2014 (VORMITTAG et al., 2014). Desses, cerca de 78\% estão localizados na Região Sudeste, sendo São Paulo o Estado com maior número de estações. Isso justifica a escolha dessa unidade geográfica para análise. Além disso, o Estado possui uma das melhores coberturas de informação de mortalidade no país. Em 2012, o sub-registro de óbitos foi de 0,5\% a partir de estimativas feitas por Szwarcwald et al. (2011). 0 percentual de causas mal definidas de internações hospitalares também se reduziu (de 2,1\% em 2007 para 1,62\% em 2014), o que contribui para a confiabilidade das estimativas.

Uma das principais limitações do estudo refere-se à possível subestimação do número de mortes projetado, pois, ao se comparar as projeções de mortalidade realizadas pelo IBGE (2013) com os valores registrados em 2012, verifica-se uma diferença de $10 \%$ a mais comparando o número efetivamente registrado e o projetado, mas que não alteraria significativamente os resultados. Em 2012, registrou-se um total de 270.432 óbitos, no Estado de São Paulo, segundo os dados do Sistema de Informações de Mortalidade, enquanto os projetados pelo IBGE foram 244.005. Pode-se, portanto, pensar nas estimativas apresentadas na Tabela 1 como um montante mínimo de óbitos decorrentes da poluição no Estado.

Vale ressaltar, ainda, que não foram consideradas mudanças nas taxas de mortalidade e de internação, o que poderia afetar os resultados. Porém, o trabalho focalizou-se, principalmente, nos cenários de poluição e seus efeitos, considerando o tamanho e a estrutura etária populacional no futuro, que possuem grande influência sobre o montante total de desfechos desfavoráveis em saúde. Por fim, foi utilizada a média dos níveis de poluição para todo o Estado de São Paulo, e não os valores referentes a cada estação de 
monitoramento para os municípios que monitoram a poluição. Entretanto, como informado na seção de metodologia, o desvio-padrão da poluição apresenta valores baixos para as 40 estações monitoradas em 2011 (baseline do estudo) e tem representatividade de municípios com alto índice de atividade industrial, grande contingente populacional e municípios de menor porte (cerca de 80 mil habitantes). Apesar dessa limitação, o estudo tem o objetivo de identificar a mortalidade e morbidade a que a população estaria submetida caso todos os habitantes fossem expostos aos níveis de poluição médios do Estado. 0 nível médio de poluição também é utilizado em estudos da OMS para estimativas da carga de doenças atribuíveis à poluição mesmo em países com poucas estações de monitoramento (WHO, 2006).

\section{Considerações finais}

Esse trabalho teve como objetivo apresentar estimativas do efeito da poluição sobre a saúde no Estado de São Paulo, de acordo com a metodologia da estimativa de mortes e doenças atribuíveis à poluição da OMS (WHO, 2006). Além disso, o estudo pretende contribuir para a discussão dos efeitos nocivos de agentes poluentes causados, principalmente, por veículos automotores, para chamar a atenção da opinião da sociedade, gestores públicos e empresários em busca de alternativas de mobilidade urbana, inovações na gestão de transportes e materiais, bem como legislação mais severa e aumento do número de estações de monitoramento da poluição. Tais medidas serviriam como guia para a implementação de ações locais que resultem em melhoria da qualidade do ar no país. Mais especificamente, estudos como esse mostram que um tema transversal tão importante como a poluição atmosférica deveria embasar discussões intersetoriais entre saúde e meio ambiente nas políticas públicas e planos de gestão para redução de emissão de poluentes, principalmente nos grandes centros urbanos, como é o caso da Região Metropolitana de São Paulo.

De forma mais específica, políticas de redução e regulação de emissões de poluentes em veículos motores, bem como incentivos a energias mais limpas de transporte, mitigariam o impacto sobre a saúde. A experiência de técnicos ambientais e estudos de impacto sobre a saúde, como o presente trabalho, servem como instrumentos de informação baseada em evidências à sociedade civil e auxílio aos planejadores para definição de novos critérios de controle da poluição.

É a partir dos padrões de qualidade do ar que se faz o diagnóstico dos níveis de poluentes que trazem malefícios para a saúde e que possibilitam determinar planos de gestão para a diminuição ou o combate à emissão de poluentes. A indefinição de novos padrões em âmbito nacional e a falta de um controle mais rigoroso dos níveis de poluição apenas protelam medidas efetivas para o combate da poluição atmosférica por fontes automotoras e fixas no país, contribuindo para a continuidade de mortes e internações em decorrência dessas causas. 


\section{Referências}

ANDERSON, H. R. et al. Meta-analysis of time-series studies and panel studies of Particulate Matter (PM) and Ozone (03). Copenhagen: WHO, 2004.

ANDRÉ, P. A. et al. Lean diesel technology and human health: a case study in six Brazilian metropolitan regions. Clinics, v. 67, n. 6, p. 639-645, 2012.

APTE, J. S.; MARSHALL, J. D.; COHEN, A. J.; BRAUER, M. Adressing global mortality from ambient $\mathrm{MP}_{2,5}$. Environmental Science \& Technology, n. 49, p. 8057-8066, 2015.

BELL, M. L. et al. The avoidable health effects of air pollution in three Latin American cities: Santiago, São Paulo, and Mexico City. Environmental Research, n. 100, p. 431-440, 2006.

BENTAYEB, M. et al. Adverse respiratory effects of outdoor air pollution in the elderly. The International Journal of Tuberculosis and Lung Disease (IJTLD), v. 16, n. 9, p. 1149-61, Sep. 2012.

BENMARHNIA, T. et al. Addressing equity in interventions to reduce air pollution in urban areas: a systematic review. Int. J. Public Health, v. 59, n. 6, p. 933-944, 2014.

BRAGA, A. L. et al. Health effects of air pollution exposure on children and adolescents in São Paulo, Brazil. Pediatric Pulmonology, v. 31, n. 2, p. 106-133, 2001.

BRASIL. Conselho Nacional do Meio Ambiente - Conama. Resolução CONAMA n. 3 de 28 de junho de 1990. Diário Oficial da União, 22/08/1990. Disponivel em: < http://www.mma.gov.br/ port/conama/legiabre.cfm?codlegi=100>.

BRUNEKREEF, B.; HOLGATE, S. T. Air pollution and health. The Lancet, v. 360, p. 1233-1242, Oct. 2002.

CANÇADO, E. D. et al. The impact of sugar cane - burning emissions on the respiratory system of children and the elderly. Environmental Health Perspectives, v. 114, n. 5, p. 725, 2006.

CAREY, I. M. et al. Mortality associations with long-term exposure to outdoor air pollution in a national English cohort. American Journal of Respiratory and Critical Care Medicine, v. 187, n. 11, p. 1226-1233, 2013.

CETESB - Companhia Ambiental do Estado de São Paulo. Qualidade do ar no estado de São Paulo 2013. São Paulo, 2014.

CONCEIÇÃO, G. M. S. et al. Air pollution and child mortality: a time-series study in São Paulo, Brazil. Environmental Health Perspectives, v. 109, suppl. 3, p. 347-350, 2001.

CONSEMA - Conselho Estadual do Meio Ambiente. Grupo de Trabalho Institucional.Revisão dos padrões de qualidade do ar e aprimoramento da gestão integrada da qualidade do ar no Estado de São Paulo. 2010. Disponível em: 〈http://www.cetesb.sp.gov.br/tecnologia/camaras/gt_ar/ RelatorioFinal-GT-NOV2010/Relat\%C3\%B3rio\%20GT\%20Final.pdf〉. Acesso em: 25 jul. 2015.

DENATRAN - Departamento Nacional de Trânsito. Frota de veículos, 2014. Disponível em: «http:// www.denatran.gov.br/frota.htm〉. Acesso em: 27 maio 2014.

DOCKERY, D. W. et al. An association between air pollution and mortality in six U.S. cities. The New England Journal of Medicine, v. 329, n. 24, p. 1753-1759, 1993.

DOMINICI, F. et al. On generalized additive models in time series studies of air pollution and health. Am J Epidemiol, v. 156, p. 193-203, 2002.

EPA - U.S. Environmental Protection Agency. EPA revises the national ambient air quality standards for particle pollution. 2012. Disponivel em: 〈http://www.epa.gov/airquality/ particulatematter/actions.html\#dec12>. 
17 set. 2014.

Value of statistical life. 2013. Disponivel em: 〈http://goo.gl/br9WwS〉. Acesso em:

FARHAT, S. C. L. et al. Effect of air pollution on pediatric respiratory emergency room visits and hospital admissions. Brazilian Journal of Medical and Biological Research, v. 38, n. 2, p. $227-$ 235, 2005.

FLACHS, E. M. et al. Population dynamics and air pollution: the impact of demographics on health impact assessment of air pollution. Journal of Environmental and Public Health, 12p. 2013.

HAMRA, G. B. et al. Outdoor particulate matter exposure and lung cancer: a systematic review and meta-analysis. Environmental Health Perspectives. National Institute of Environmental Health Sciences, 2014. Disponivel em: 〈http://dx.doi.org/10.1289/ehp.1408092〉. Acesso em: 29 jun. 2015.

IARC - International Agency for Research on Cancer. Outdoor air pollution a leading environmental cause of cancer deaths. Lyon: WHO, 2013. Disponivel em: 〈http://www.iarc.fr/en/media-centre/ iarcnews/pdf/pr221_E.pdf. Acesso em: 10 out. 2014.

IBGE - Instituto Brasileiro de Geografia e Estatística. Projeção da população das unidades da federação por sexo e idade: 2000-2030. Rio de Janeiro, 2013.

IHME. Global Burden of Disease Study 2010 (GBD 2010). Population estimates 1970-2010. 2012. Disponivel em: <http://ghdx.healthmetricsandevaluation.org/record/global-burden-diseasestudy-2010-gbd-2010-population-estimates-1970-2010〉. Acesso em: 25 jul. 2015.

LADEN, F. et al. Reduction in fine particulate air pollution and mortality. American Journal of Respiratory and Critical Care Medicine, v. 173, n. 6, p. 667-672, 2006.

LELIEVELD, J.; EVANS, J. S.; FNAIS, M.; GIANNADAKI, D.; POZZER, A. The contribution of outdoor air pollution sources to premature mortality on a global scale. Research letter. Nature, n. 525 , p. 367-384, 2015.

LEPEULE, J. et al. Chronic exposure to fine particles and mortality: an extended follow-up of the Harvard six cities study from 1974 to 2009. Environmental Health Perspectives, v. 120, n. 7 , p. 965-970, 2012.

LIN, C. A. et al. Air pollution and neonatal deaths in São Paulo, Brazil. Brazilian Journal of Medical and Biological Research, v. 37, n. 5, p. 765-70, 2004.

LIPFERT, F. W. Air pollution and mortality: specification searches using SMSA-based data. Journal of Environmental and Economy Management, v. 11, n. 3, p. 208-243, 1984.

McCORMACK, M. C. et al. Indoor particulate matter increases asthma morbidity in children with non-atopic and atopic asthma. Annals of Allergy, Asthma \& Immunology, v. 106, n. 4, p. 308315, April 2011.

MEDEIROS, A. et al. Relationship between low bithweight and air pollution in the city of São Paulo, Brazil. Public Health Magazine, v. 39, n. 6, p. 965-972, 2005.

NASCIMENTO, L. F. C. et al. Efeitos da poluição atmosférica na saúde infantil em São José dos Campos, SP. Revista de Saúde Pública, v. 40, n. 1, p. 77-82, 2006.

NIELSEN, O. R. et al. Air pollution and lung cancer incidence in 17 European cohorts: prospective analyses from the European Study of Cohorts for Air Pollution Effects (ESCAPE). The Lancet Oncology, v. 14, n. 9, p. 813- 22, Jul. 2013.

OSTRO, B. Outdoor air pollution: assessing the environmental burden of disease at national and local levels. Geneva: World Health Organization, 2004 (Environmental Burden of Disease 
Series, n. 5). Disponível em: 〈http://www.who.int/quantifying_ehimpacts/ publications/ebd5. pdf〉. Acesso em: 20 jul. 2015.

PEREIRA, L. A. et al. Association between air pollution and intrauterine mortality in São Paulo, Brazil. Environmental Health Perspectives, v. 106, n. 6, p. 325-329, 1998.

PETERS, A. Particulate matter and heart disease: Evidence from epidemiological studies. Toxicology and Applied Pharmacology, v. 207, suppl. 2, p. 477-482, 2005.

POPE, C. A. et al. Cardiovascular mortality and long-term exposure to particulate air pollution: epidemiological evidence of general pathophysiological pathways of disease. Circulation, v. 109, n. 1, p. 71-77, 2004.

. Lung cancer, cardiopulmonar mortalilty, and long-term exposure to fine particulate air pollution. Journal of the American Medical Association, v. 287, n. 9, p. 1132-1141, 2002.

Particulate air pollution as a predictor of mortality in a prospective study of US adults. American Journal of Respiratory and Critical Care Medicine, v. 151, n. 3, p. 669-674, 1995.

POPE, C. A.; DOCKERY, D. W. Health effects of fine particulate air pollution: lines that connect. Air \& Waste Management Association, v. 56, n. 6, p. 709-742, 2006.

RITZ, B.; WILHELM, M.; ZHAO, Y. Air pollution and infant death in Southern California, 1989-2000. Pediatrics, v. 118, n. 2, p. 493-502, 2006.

RODRIGUES, C. G.; ANDRADE, M. V.; QUEIROZ, B. L.; MACHADO, C. J. The applicability of the LeeCarter method to forecast health services use in Brazil. In: HOQUE, N.; McGEHEE, M. A.; BRADSHAW, B. Applied demography and public health. Springer, Applied Demographic Series, v. 3, 2013.

SALDIVA, P. H. N. et al. Air pollution and mortality in elderly people: a time-series study in São Paulo, Brazil. Arch Environ Health, v. 50, n. 2, p. 159-163, 1995.

SOFER, T. et al. Exposure to airborne particulate matter is associated with methylation pattern in the asthma pathway. Epigenomics, v. 5, n. 2, p. 147-154, April 2013.

STRUNK, B. C.; GINSBURG, P. B.; BANKER, M. I. The effect of population aging on future hospital demand. Health Affairs (Millwood), n. 25, p. 141-149, 2006.

SZWARCWALD, C. L.; MORAIS-NETO, O. L.; FRIAS, P. G.; SOUZA JUNIOR, P. R. B.; ESCALANTE, J. J. C.; LIMA, R. B., VIOLA, R. C. Busca ativa de óbitos e nascimentos no Nordeste e na Amazônia Legal: estimação das coberturas do SIM e do Sinasc nos municípios brasileiros. In: BRASIL. Ministério da Saúde. Secretaria de Vigilância em Saúde. Departamento de Análise de Situação em Saúde. Saúde Brasil 2010: uma análise da situação de saúde e de evidências selecionadas de impacto de ações de vigilância em saúde. Brasília (DF): MS, 2011. p. 79-98.

ULIRSCH, G. V. et al. Effect of particulate matter air pollution on hospital admissions and medical visits for lung and heart disease in two southeast Idaho cities. Journal of Exposure Science and Environmental Epidemiology, n. 17, p. 478-487, 2007.

VAHLSING, C.; SMITH. K. R. Global review of national ambient air quality standards for MP10 and SO2 (24h). Air Quality, Atmosphere \& Health, v. 5, n. 4, p. 393-399, 2012.

VORMITTAG, E. M. P. A. A.; COSTA, R. R.; BRAGA, A. A.; MIRANDA, M. J.; NASCIMENTO, N. C.; SALDIVA, P. H. Monitoramento da qualidade do ar no Brasil. Instituto Saúde e Sustentabilidade, 2014. Disponivel em: 〈http://goo.gl/7Xvu2i〉. Acesso em: 26 jul. 2015.

WELLENIUS, G. A.; SCHWARTZ, J.; MITTLEMAN, M. A. Particulate air pollution and hospital admissions for congestive heart failure in seven United States cities. American Journal of Cardiology, v. 97, n. 3, p. 404-408, 2006. 
WHO - World Health Organization. Air quality guidelines - Global Update 2005. Copenhagen: WHO, 2006. Disponível em: 〈http://goo.gl/XQRNLZ〉. Acesso em: 10 out. 2014.

Health and the environment: addressing the health impact of air pollution. Draft resolution proposed by the delegations of Albania, Chile, Colombia, France, Germany, Monaco, Norway, Panama, Sweden, Switzerland, Ukraine, United States of America, Uruguay and Zambia. Sixty-Eighth World Health Assembly. Agenda item 14.6. A68/CONF./2 Rev.1. 26 May 2015.

\title{
Sobre os autores
}

Cristina Guimarães Rodrigues é demógrafa, doutora em Demografia pelo Centro de Desenvolvimento e Planejamento Regional - Cedeplar, da Universidade Federal de Minas Gerais - UFMG. Professora de Demografia na Fundação Instituto de Pesquisas Contábeis, Atuariais e Financeiras - Fipecafi.

Evangelina da Motta Pacheco Araújo Vormittag é médica, doutora em Patologia pela Faculdade de Medicina da Universidade de São Paulo - USP. Diretora do Instituto Saúde e Sustentabilidade.

Julia Affonso Cavalcante é gestora ambiental, mestranda em Geografia Humana pela Faculdade de Filosofia, Letras e Ciências Humanas da Universidade de São Paulo - USP.

Paulo Hilário Nascimento Saldiva é médico, doutor em Patologia pela Universidade de São Paulo - USP. Professor titular de Patologia na Faculdade de Medicina da Universidade de São Paulo - USP.

\section{Endereço para correspondência}

\author{
Cristina Guimarães Rodrigues \\ Alameda Fernão Cardim, 102 apto. 51, Jardim Paulista \\ 01403-020 - São Paulo-SP, Brasil \\ Evangelina da Motta Pacheco Araújo Vormittag \\ Rua Brigadeiro Luís Antônio, 278 - 7ํandar \\ 01318-901 - São Paulo-SP, Brasil \\ Julia Affonso Cavalcante \\ Avenida Professor Afonso Bovero, 918 apto. 185, Perdizes \\ 05019-010 - São Paulo-SP, Brasil \\ Paulo Hilário Nascimento Saldiva \\ Avenida Dr. Arnaldo, 455 sala 1103 \\ 01245-903 - São Paulo-SP, Brasil
}

\section{Abstract}

Forecasting of mortality and hospital admissions from public healthcare attributable to air pollution in State of São Paulo between 2011 and 2030

National air quality standards in Brazil were established in the 1990s and are now outdated in relation to international patterns. This creates a challenge for effective control of air pollution levels and contributes to increasing mortality and hospital admissions rates. In order to quantify the impact of adverse health outcomes due to the concentration of particulate matter in São Paulo, this paper aims to project the number of deaths, hospitalizations and amounts spent on 
public hospital admissions from 2012 to 2030. It considers distinct scenarios of air pollution by fine particulate matter (PM2.5), using the year 2011 as baseline. The results show that if air pollution continues at the current levels, it will cause more than 246 thousand deaths from all causes between 2011 and 2030, as well as some 953 thousand hospital admissions and an estimated public health cost of $R \$ 1.6$ billion due to hospitalizations. The magnitude of these results demonstrates the need to implement more rigorous measures to control air pollution and to encourage clean energy transportation, among other public actions, in order to reduce damage to the health of the population and to diminish government spending.

Keywords: Air pollution. Public health. Sao Paulo State. Particulate matter.

\section{Resumen}

Proyección de la mortalidad y las admisiones hospitalarias en la red pública de salud atribuibles a la contaminación atmosférica en el Estado de São Paulo entre 2011 y 2030

En Brasil, los estándares nacionales de calidad del aire datan de la década de 1990, es decir, están obsoletos en comparación con las normas internacionales, lo que dificulta un control eficaz de los niveles de contaminación y contribuye a la continuidad de las muertes y hospitalizaciones ocasionadas por esta razón. Con el fin de cuantificar el total de los resultados adversos para la salud debidas a la concentración de material particulado en el Estado de São Paulo, este trabajo tiene como objetivo realizar proyecciones de muertes, hospitalizaciones y gastos por internaciones en hospitales públicos atribuibles a la polución atmosférica en el período comprendido entre 2012 y 2030. Para ello se han considerado distintos escenarios de contaminación por material particulado fino (MP2,5), tomando como referencia el año base 2011. Los resultados muestran que, manteniendo el mismo nivel de contaminación observado en esa fecha, habrá un total de más de 246.000 muertes por todas las causas entre 2011 y 2030, alrededor de 953.000 ingresos hospitalarios en el sistema público de salud y un gasto público por internaciones estimado en más de $R \$ 1,6$ billones. La magnitud de los resultados pone en evidencia la necesidad de implementar medidas más rigurosas para el control de la contaminación del aire, formas alternativas de transporte con energía limpia, entre otras acciones, con el fin de reducir los daños a la salud de la población y los gastos del gobierno.

Palabras clave: Contaminación del aire. Salud pública. Estado de São Paulo. Material particulado. 